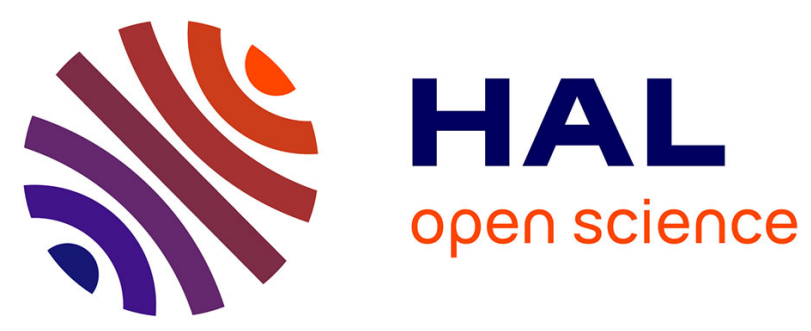

\title{
Closed-Loop Control of the Centre of Pressure in Post-Stroke Patients With Balance Impairments
}

Claire Kemlin, Fabien Vérité, Veronique Marchand-Pauvert, Pierre-François

Pradat, Pascale Pradat-Diehl, Alain Giron, Wael Bachta

\section{To cite this version:}

Claire Kemlin, Fabien Vérité, Veronique Marchand-Pauvert, Pierre-François Pradat, Pascale PradatDiehl, et al.. Closed-Loop Control of the Centre of Pressure in Post-Stroke Patients With Balance Impairments. IEEE Transactions on Neural Systems and Rehabilitation Engineering, 2019, 27 (2), pp.265-274. 10.1109/TNSRE.2019.2892177 . hal-02492096

\section{HAL Id: hal-02492096 https://hal.sorbonne-universite.fr/hal-02492096}

Submitted on 26 Feb 2020

HAL is a multi-disciplinary open access archive for the deposit and dissemination of scientific research documents, whether they are published or not. The documents may come from teaching and research institutions in France or abroad, or from public or private research centers.
L'archive ouverte pluridisciplinaire HAL, est destinée au dépôt et à la diffusion de documents scientifiques de niveau recherche, publiés ou non, émanant des établissements d'enseignement et de recherche français ou étrangers, des laboratoires publics ou privés. 


\title{
Closed-loop control of the centre of pressure in post-stroke patients with balance impairments
}

\author{
Claire Kemlin, Fabien Vérité, Véronique Marchand-Pauvert, Pierre-François Pradat, Pascale Pradat-Diehl, \\ Alain Giron and Wael Bachta
}

\begin{abstract}
When a lightly touched surface is moved according to a closed-loop control law, it has been shown in young adults that the Centre of Pressure (CoP) can be displaced in a controllable way without the conscious cooperation of participants. In this closed-loop paradigm, the surface velocity was continuously adjusted according to the CoP position. Since the closed-loop control of the CoP does not require the participant's voluntary cooperation, it could be of interest for the development of innovative biofeedback devices in balance rehabilitation. Before anticipating the implementation of this closed-loop control paradigm with patients, it is necessary to establish its effects on people suffering from balance impairments. The aim of this study was to assess the effects of this CoP closed-loop control in poststroke (PS) patients and aged-matched healthy controls. Efficacy of the closed-loop control for driving the patients' CoP was assessed using the saturation time and two scores computing the error between the predefined and the current CoP trajectories. $68 \%$ and $83 \%$ of the trials were considered as successful in patients and controls, respectively. The global tracking error of the closed-loop score was similar between the two groups. However, when examining the real $\mathrm{CoP}$ displacement from the starting position to the desired one, PS patients responded to the closed-loop control to a lesser extent than controls. These results, obtained in the same conditions for healthy and post-stroke individuals could be improved by tuning the closed-loop parameters according to individual characteristics. This study paves the road towards the development of involuntary/automatic biofeedback techniques in more ecological conditions.
\end{abstract}

Index Terms-balance, biofeedback, centre of pressure, closedloop control, light touch. ${ }^{1}$

\section{InTRODUCTION}

Loss of postural control leads to an increased incidence of falls and represents a major health issue. Natural aging processes [1] as well as neurogical disease (e.g. stroke) [2] may alter a person's ability to effectively regulate standing postures. In order to restore adaptive postural control, patients may undergo rehabilitation programs, which consist of

Manuscript submitted for review September $7^{\text {th }}, 2018$. This work was funded by the ANR within the Investissements d'Avenir, programme under reference ANR-11-IDEX-0004-02 and within the project i-Gait, under reference ANR16-CE33-0012.

C. Kemlin is with Sorbonne Université, UPMC Université Paris 06, CNRS UMR 7225, Inserm U1 127, Institut du Cerveau et de la Moelle (ICM), F-75013, Paris, France (e-mail : claire.kemlin@gmail.com).

F. Vérité is with Aix Marseille Université, CNRS UMR 7287, Institut des Sciences du Mouvement (ISM), Marseille, France (email : fabienverite@gmail.com).

V. Marchand-Pauvert and A. Giron are with Sorbonne Université, UPMC Université Paris 06, INSERM UMR-S 1146, CNRS UMR 7371, Laboratoire intensive sets of physical exercises involving balance tasks [3], [4]. In addition to conventional clinical balance scales [5], force plates can be used to monitor body sway [6] and provide a quantified assessment of postural control [7], [8].

\section{A. Traditional sensory biofeedback requires users' voluntary action}

Rehabilitation programs may benefit from sensory biofeedback techniques, in which the central nervous system (CNS) is supplied with relevant additional sensory information [9]. Providing sensory biofeedback represents an effective approach for improving balance after stroke [10]-[12]. Sensory biofeedback systems are usually composed of (1) a balance assessment device, (2) an algorithm extracting meaningful postural data and (3) another device providing pertinent information to the participant regarding their performance. This feedback may take the form of visual [13], [14], auditory [15], vibrotactile [16]-[18] or kinesthetic [19] cues.

For instance, in [13], [14], the position of the participant's Centre of Pressure (CoP) was monitored by a Nintendo Wii Balance Board and displayed in real-time on a screen. Using this setup, patients were asked to move their CoP towards different targets in the video game interface with the objective of retraining postural control. In [15], [20], balance was monitored using an Inertial Measurement Unit (IMU) integrated in a smartphone while headphones were used to provide auditory feedback on an individual's postural activity. In [16],

d'Imagerie Biomédicale, Paris, France (e-mail: veronique.marchandpauvert@inserm.fr, giron@lib.upmc.fr).

P.F. Pradat is with AP-HP, Pitié-Salpêtrière Hospital, Neurology Department, Paris, France (e-mail: pierre-francois.pradat@aphp.fr).

P. Pradat-Diehl is with AP-HP, Pitié-Salpêtrière Hospital, Physical Medecine and Rehabilitation Department, Paris, France (e-mail : pascale.pradat@aphp.fr).

W. Bachta is with Sorbonne Université, UPMC Université Paris 06, CNRS UMR 7222, INSERM U1150, Institut des systèmes intelligents et de robotique (ISIR), Paris, France (e-mail : fabienverite@gmail.com, bachta@isir.upmc.fr). 
[17], balance was similarly monitored using an IMU and a wobble board. In this case, participants received oriented warning messages from two vibrotactors attached to the waist. In [18], post-stroke patients with hemiparesis used a biofeedback-based device for the purpose of improving gait. This system was composed of a smartphone for balance measurements, a kinesthetic haptic cane held by the non-paretic hand, and a set of vibrotactile actuators attached to the paretic lower limb. The system was effective for increasing the walking speed by improving the active involvement of the paretic limb. In [19], an IMU and an Phantom Omni ${ }^{\circledR}$ were used to quantify postural sway and provide light directional forces indicating how the body should tilt. The authors reported positive effects in poststroke patients.

Furthermore, combining several sensory cues may potentially enhance balance training outcomes. Indeed, certain projects have specifically focused upon the development of multimodal sensory feedback exercises. For instance, in [21], visual and vibrotactile feedback was provided simultaneously to patients with Parkinson's disease using a smartphone and a belt housing a processing unit, miniaturized sensors, and vibrating actuators. Of course, the means by which the sensory information is coded is also a key factor. Ongoing investigations are required to identify those which are easiest for the individual to interpret [22].

Importantly though, all the biofeedback-based systems described above require the voluntary cooperation of each participant. Subjects must perceive and interpret the supplementary information which is transmitted before responding through postural adjustments. Having biofeedback-based systems that function with no or minimal active involvement of the user could be particularly useful in neurorehabilitation, especially for patients with important cognitive impairments. For such patients, dealing with several tasks simultaneously is very challenging and providing this type of feedback may help them to improve their posture implicitly. It could also be interesting to study the short and longterm effects of repeated sessions of this unconscious feedback on natural postural sway. Further technological development might also allow this type of approach to be adapted in order to implant unconscious feedback in technical walking aids.
B. Towards alternative sensory biofeedback with implicit, closed-loop control of the CoP

In [23], it was reported that lightly touching a stationary surface with the forefinger reduced postural sway with eyes either closed or open, and without the participants' voluntary cooperation. The stabilizing effect of light touch is effective in healthy participants, and has also been verified in patients with neurological disorders [24] such as post-stroke patients [25], [26]. Given that the forces involved in the light touch paradigm are less than $1 \mathrm{~N}$, the postural changes observed are not a consequence of increased mechanical support. Rather, the stabilizing effects of the light touch correspond with the supplementary sensory information provided to the CNS. The motionless light touch paradigm explains, for instance, how some patients benefit from a cane [27], [28] or a walker [29] even with small interaction forces between the user and the technical aid.

Following from these findings, a portable sensory augmentation device has been developed to help healthy subjects to correct their posture with an IMU monitoring balance and a skin-stretch motor providing tactile information to the forefinger [30]. Participants were not aware of the role of the skin stretcher. When the participant leaned forward, the fingertip was stretched backward and the participant was expected to move backward in reaction without interpreting the signal or making any decision. While the efficacy of this tactile-based system is not yet clear in healthy participants, its effects when simulating sensory deficits appear promising. In [31]-[33], it was reported that lightly touching a sinusoidally moving surface induces $\mathrm{CoP}$ displacements with the same frequency when participants' eyes are either closed [31], or open [33]. This coupling occurs without any voluntary participation of participants. Often, participants were not even aware of the surface motion.

Based on these findings, we have developed a system for closed-loop control of the CoP [34], [35] where the sagittal position of the CoP was servo-controlled around a smooth trajectory in order to bring it to a new arbitrary, predefined target position. In this closed-loop control, the lightly touched surface velocity was adjusted according to the difference between the desired (i.e. the arbitrary predefined 
target) $\mathrm{CoP}$ position and the actual participant's $\mathrm{CoP}$ position at each sampling time.

\section{Contributions and outline:}

Our long-term objective is to develop a sensory biofeedback-based device for improving balance and mobility in rehabilitation settings. Our system, based on closed-loop control of participants' CoP, does not require any voluntary involvement of participants.

Before anticipating any implementation of the closed-loop control of the $\mathrm{CoP}$ in rehabilitation, it is necessary to validate its efficacy in patients. To our best knowledge, our study is the first to include patients in an experiment aiming at displacing their CoP using a moving, lightly touched surface.

Our study has three objectives. The first is to assess the possibility of driving participants' $\mathrm{CoP}$ both forward and backward, using a closed-loop and a new smooth reference trajectory. The second objective is to introduce two new evaluation criteria: (1) the saturation time, denoted $\Delta t_{\text {sat }}$ measured the amount of time during which the closed-loop failed to drive the CoP position, a situation which occurs when the moving surface reaches the limits of its working space. If this saturation time is excessively long, one may conclude that the trial is unsuccessful. (2) $e_{\text {start }}$ which gave information concerning the displacement achieved by the CoP. This score indicated the difference between the $\mathrm{CoP}$ displacement decided by the experimenter $(8 \mathrm{~mm})$ and that which was actually achieved by the participant. The third objective is to assess and compare the efficacy of the CoP closed-loop control in Post-Stroke (PS) patients and aged-matched healthy subjects by using the tracking error $C$, already published in [34], [35]. $e_{\text {start }}$, along with $C$ was used to quantify the efficacy of the closed-loop control and to compare the results achieved in controls and post-stroke patients.

The paper is organized as follows. The following section is dedicated to the presentation of the device, the closed-loop control of the CoP, the tracking error score $C$, and the two new evaluation criteria $\left(\Delta t_{\text {sat }}\right.$ and $\left.e_{\text {start }}\right)$. The third section then presents the experimental methods while the fourth section introduces the commented results. Finally, and before concluding, a general discussion about our results, the limitations of the study and the ongoing investigations are given.

\section{MATERIAL AND SCORES ASSESSING THE CLOSED-} LOOP EFFECTS

\section{A. The experimental setup}

The experimental setup, depicted in Fig. 1, was composed of a force plate (AMTI BP400600-1000, Watertown, USA), its associated amplifier (AMTI mini amp, Watertown, USA), and a custom-made translational motorised device which allowed for the displacement of the surface on which participants placed their forefinger. The motorised device was a belt-pulley mechanism driven by a direct current electric motor. The speed of the belt was controlled by an Elmo Solo-Whistle servo-drive. The belt was equipped with a flexiforce sensor in order to monitor the force applied by the participants' forefinger. A double-sided adhesive tape was stuck on the top of the flexiforce sensor. The belt and the finger motions were equal. The equipment was non-reversible, i.e. it could not be displaced by the finger motion. The belt could move $20 \mathrm{~mm}$ backward and $20 \mathrm{~mm}$ forward around the initial position. The working space length was equal to $40 \mathrm{~mm}$.

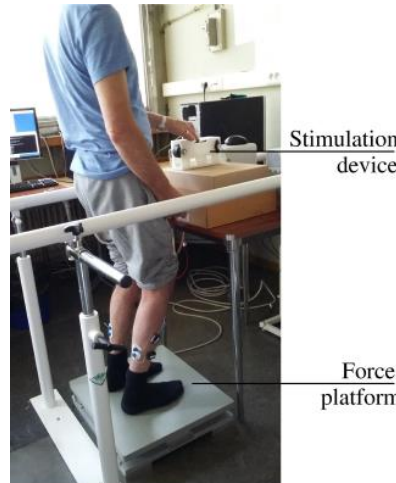

(a)

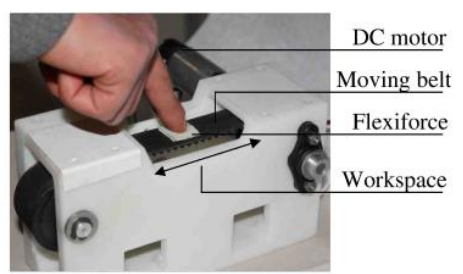

(b)
Figure 1: Experimental setup. (a) Global view of the setup during a trial with a post-stroke participant with a right-sided hemiparesis and (b) close view of the translational motorized device.

At the beginning of each closed-loop trial, the flexiforce was brought back to the center of the working space. A computer equipped with an acquisition board ran custom software, which acquired the force plate measurements, computed the $\mathrm{CoP}$ position, and sent the reference speed to the motor servo-drive at a rate of $500 \mathrm{~Hz}$. Loudspeakers in the room emitted pink noise to prevent the sound generated by the motor from giving acoustic cues to participants about the belt motion. 


\section{B. Closed-loop control of the CoP position}

Our closed-loop paradigm aimed at displacing the $\mathrm{CoP}$ towards a new position. This was achieved by servoing the CoP position at each sampling time around an arbitrary smooth reference trajectory denoted $p^{*}(t)$ (illustrated in blue in Fig. 2(b) and 3). Unlike [31], [32], the lightly touched surface did not move independently. The belt velocity was set according to the instantaneous $\mathrm{CoP}$ position as in [34], [35]. More precisely, at each sampling time, the belt velocity (equal to the finger velocity) was set to be proportional to the error between the desired position of the reference trajectory and the participant's filtered position as illustrated in Fig 2(a) and shown in the following equation:

$$
v_{b}(t)=K\left(p^{*}(t)-\widetilde{p}(t)\right)
$$

where $v_{b}(t)$ is the speed of the belt at some time $(t)$, $K$ is a constant feedback gain, $p^{*}(t)$ is the reference trajectory and can be seen as the desired CoP position, $p(t)$ is the current CoP position in the anteroposterior direction, $\widetilde{p}(t)$ is a low-pass filtered version of $p(t)$. The low-pass filtering was applied using a first order Butterworth filter with a cutoff frequency of $0.3 \mathrm{~Hz}$. This filtering enabled us to retain the slow component of each participant's postural sway. The feedback gain was tuned in a trial-and-error fashion in a pilot study on young and healthy subjects not documented here and set to 0.48 $\mathrm{s}^{-1}$.

(a)

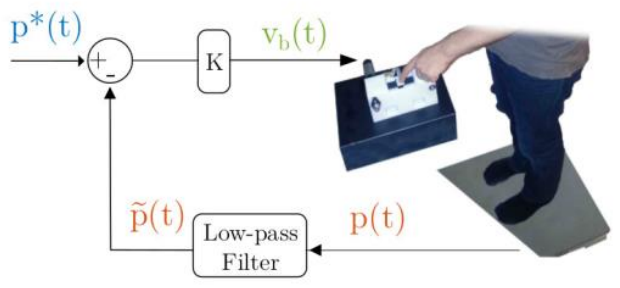

(b)

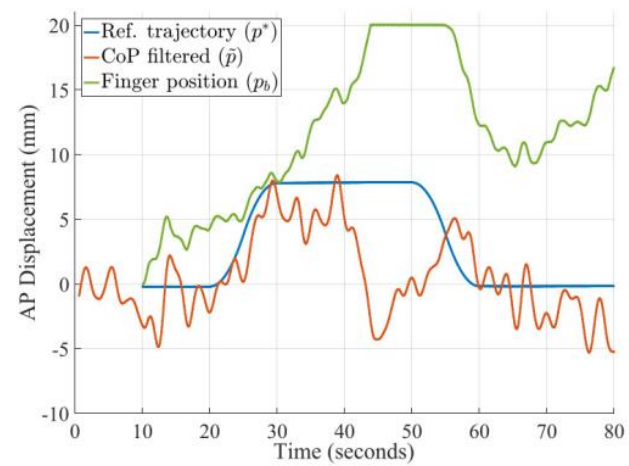

Figure 2: Closed-loop control of the CoP using moving light-touch. In blue: the reference trajectory, in red: the actual trajectory of the CoP and in green: the belt/finger displacements. (a) the closed-loop control law, (b) performance of one post-stroke patient during one forward closed-loop trial.

With this experimental closed-loop setup, if the participants leaned forwards and overreached the desired value of the reference trajectory (i.e. $\widetilde{p}>p^{*}$ ), the belt moved backwards in order to drive the current CoP position towards the reference value $p^{*}$. Inversely, if the participants leaned backwards and under-reached the desired value (i.e. $\widetilde{p}<p^{*}$ ), the belt moved forwards.

The reference trajectory has been defined to avoid sharp transitions like the one reported in [34]. Our trajectory was intended to guide the $\mathrm{CoP}$ towards a new reference position $(8 \mathrm{~mm}$ forward and backward) implicitly (i.e. without the conscious cooperation of the participants). The aimed displacement length of $8 \mathrm{~mm}$ was chosen for two reasons. First, the displacement had to be large enough to observe a clear shift in participants' mean $\mathrm{CoP}$ position in the AP direction. For participants lightly touching a stable surface with closed eyes, the mean sway amplitude in the AP direction was 2.5 $\mathrm{mm}$ in older healthy participants (60-86 years old) [36] and around $2 \mathrm{~mm}$ in post-stroke participants [25]. Thus, the $8 \mathrm{~mm}$ value has been chosen to be distinctly higher than these values. Also, the aimed displacement could not be excessively large or at a point where the safety and postural stability of the patients would be compromised.

The reference trajectory can either present a forward and then backward direction in the forward closedloop condition (F_CL). It can also achieve the reverse path, i.e. a posterior motion followed by an anterior one in the backward closed-loop condition (B CL).

The time intervals of the reference trajectory $p *$ were defined over the 80 -second trial as follows:

[0 to 10]s: The mean CoP position in the AP direction was computed during the first 10 seconds.

[10 to 20]s: $p^{*}$ was set to be equal to the mean CoP position calculated during the first 10 seconds.

[20 to 30] s: $p^{*}(t)$ was a smooth trajectory moving 8 $\mathrm{mm}$ forward or backward, depending on whether a Forward Closed-loop (F_CL) or a Backward Closedloop (B CL) was implemented.

[30 to 50$] \mathrm{s}: p^{*}(t)$ remained constant at its new value ( $8 \mathrm{~mm}$ away from the initial position).

[50 to 60]s: $p^{*}(t)$ was a smooth trajectory moving 8 $\mathrm{mm}$ backward or forward, depending on whether a Forward Closed-loop (F_CL) or a Backward Closedloop (B_CL) was implemented. 
[60 to 80$] \mathrm{s}: p^{*}(t)$ was set to be equal to the mean CoP position calculated during the first 10 seconds.

The reference trajectory time intervals were the same for all closed-loop trials.

The goal of the closed-loop was thus to maintain each participant's CoP position for the first 10 seconds, then move it forward or backward $8 \mathrm{~mm}$ (in ten seconds) and maintain it at the new position for 20 seconds, then move it back to its initial position (in 10 seconds), and once again maintain it at the initial position for the last 20 seconds. Only the direction of the reference trajectory was different between the F_CL and the $\mathrm{B}$ CCL conditions. The $\mathrm{F} \_\mathrm{CL}$ and $\mathrm{B}$ - $\mathrm{CL}$ were implemented to see if there was a significant difference between these two anteroposterior directions.

Fig. 2(b) shows an example of a single forward closed-loop trial in a post-stroke patient. During this specific trial, when the filtered $\mathrm{CoP}$ was lower than the reference trajectory, the finger was moved forward $(v(t)>0$ like in the [15 to 40$] \mathrm{s}$ time interval). Conversely, when the filtered CoP was higher than the reference trajectory, the finger was moved backward $(v(t)<0$ like in the [55 to 60] sime interval). In this trial, we can observe that the closed-loop control induced the expected $\mathrm{CoP}$ displacement in the first forty seconds and then failed to drive the CoP.

\section{Closed-loop scores}

Three different scores, computed to assess the closed-loop efficacy have been compared between the two groups:

1) The absolute value of the mean tracking error, $\epsilon$, was computed as follows $\epsilon=$ $\frac{1}{N}\left|\sum p^{*}-p\right|$ where $\mathrm{N}=35000$ is the number of samples recorded during the trial. The absolute value was used because oscillations about a reference upright stance is necessary for maintaining balance. This score can be seen as the tracking error from an engineering point of view [34], [35].

2) The total duration for which the finger was in saturation during one closed-loop trial for each individual, $\Delta t_{\text {sat. }}$. At certain moments, the belt, and thus the finger, reached the chosen maximum belt displacement of 20 $\mathrm{mm}$ in either the positive or the negative direction (see e.g. [45 to 55]s interval in Fig. 2(b) in an individual trial in a stroke subject). This situation was called "saturation". This was susceptible to happen if the closed-loop failed to keep the CoP of the participant close to the desired reference trajectory. In this case, the moving surface, touched by the finger, stopped its displacement and was no longer able to adjust the CoP trajectory. This mechanism of saturation was implemented to avoid an endless displacement of the belt. For example in a F_CL trial, if the CoP remained lower than the reference trajectory, the finger velocity $v_{b}(t)$ would have remained positive, and the belt would have moved forward indefinitely. Instead, the finger stopped moving when it reached the working space upper boundary of the motorized device $(=20$ $\mathrm{mm}$ from the initial position). The belt could move again backward if the $\mathrm{CoP}$ desired position became lower than the actual $\mathrm{CoP}$ position (thanks to a postural change or a change of the CoP desired position).

If the belt and consequently the finger were in the saturation situation for a long time, we expected the closed-loop control to give poor results. The higher this score was, the less effective the closed-loop was in driving the CoP.

3) $e_{\text {start }}$ : During the [20 to 30$] \mathrm{s}$ interval of the closed-loop trials, the goal was to displace the mean $\mathrm{CoP}$ by $8 \mathrm{~mm}$ (forwards or backwards) in the AP direction. The actual displacement of the CoP mean position, denoted $\left|d_{\text {start }}\right|$, was obtained by computing the absolute value of the difference between the mean CoP positions during the [30 to 50]s and $[10$ to 20$] \mathrm{s}$ time intervals. The difference between the expected displacement $(8 \mathrm{~mm})$ and the actual displacement was denoted $e_{\text {start }}$ $=8-\left|d_{\text {start }}\right|$. Figure 3 illustrates how $e_{\text {start }}$ was computed for a trial recorded with a poststroke patient in F_CL condition. 


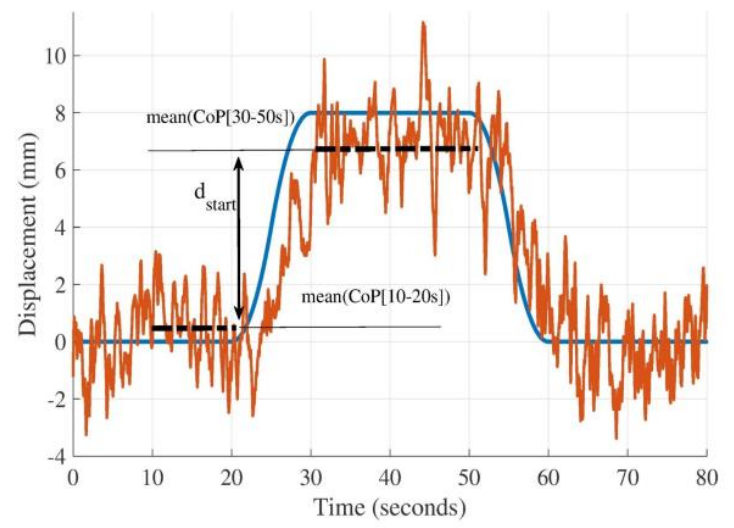

Figure 3: Calculation of the closed-loop performance score, $e_{\text {start }}$ which is the difference between $8 \mathrm{~mm}$ and the actual CoP displacement (between the [30 to $50] \mathrm{s}$ and the [10 to 20$] \mathrm{s}$ intervals). The lesser $e_{\text {start }}$ is, the more the CoP is displaced, the more efficient is the closed-loop control.

In summary, the smaller these three scores were, the better the performance was.

In order to explore more specifically the closed-loop effects, we chose to analyze trials where the finger did not reach saturation for too much time. For this purpose, $\Delta t_{\text {sat }}$ of all the participants' trials (controls and PS in both conditions) were merged in $\Delta t_{A L L}$, and only trials with saturation times less than the third quartile of $\Delta t_{A L L}$ were retained for a deeper investigation.

\section{EXPERIMENTAL METHODS}

\section{A. Participants}

Twenty PS patients were recruited at La PitiéSalpêtrière hospital from the Stroke unit and two rehabilitation departments. All PS patients were hemiparetic and presented with balance impairments (Berg balance scale $<56$ ). There were as many right sided stroke patients as left sided stroke patients. The median [Q1: first interquartile - Q3: third interquartile] time between stroke onset and inclusion was variable: 6.4 months [2.1 - 26.9]. The median [Q1 - Q3] score obtained by the PS patients for the Berg balance scale was 44.5 [42 - 48] out of 56. All patients required technical assistance to walk indoors and/or outdoors. Patients were either hospitalized for care and rehabilitation or underwent the experiment during an outpatient visit. Twenty healthy, age-matched participants were also recruited. The study was approved by the appropriate legal and ethical authority (CPP Ile de France VI Pitié-Salpêtrière - ID RCB: 2014-A0165839) in accordance with the Declaration of Helsinki. Written informed consent was obtained from all participants. Participant characteristics are given in Table I.

TABLE I: CHARACTERISTICS OF THE PARTICIPANTS.

\begin{tabular}{|c|c|c|c|}
\hline & Controls $(\mathrm{N}=\mathbf{2 0})$ & PS patients $(\mathrm{N}=20)$ & p-value \\
\hline \multicolumn{4}{|c|}{ Clinical characteristics } \\
\hline Age & $59[56-62]$ & $63[55-68]$ & NS \\
\hline $\operatorname{Sex}[\mathrm{m} / \mathrm{f}]$ & $10 / 10$ & $14 / 6$ & NS \\
\hline Height [m] & $1.73[1.64-1.79]$ & $1.67[1.63-1.74]$ & NS \\
\hline BERG & $55[55-56]$ & $45[42-48]$ & $* * *$ \\
\hline modified FAC & $8[8-8]$ & $5[5-6]$ & $* * *$ \\
\hline \multicolumn{4}{|c|}{ Posturographic parameter } \\
\hline SA (mm ) & $193.6[120.0-312.0]$ & $456.1[265.6-663.5]$ & $* *$ \\
\hline
\end{tabular}

Data are presented as median [Q1: first interquartile - Q3: third interquartile]. PS: post-stroke, FAC: Functional ambulation classification, SA: sway area, NS: non-significant, $<0.001$ : ***, $<0.01$ : **.

\section{B. Experimental procedure}

The experiment lasted around 1 hour per participant. Balance and gait autonomy were assessed with the Berg balance scale and the modified functional ambulation classification (FAC) by a physiotherapist. During the experiment, participants stood on the force plate in a bipedal stance. Feet were positioned at hip width. For each participant, the position of the feet was marked during the first trial and reproduced for the following trials. If participants were unable to place their feet as indicated by the markers, they received help from the experimenter. Participants had the possibility to sit in order to rest between trials at any point during the experiment. The motorized device was placed on a table in front of the participant so as to move the belt in the anteroposterior (AP) direction. The height was adjusted to be approximatively level with the participant's centre of mass (between the pubis and the navel). As illustrated in Fig. 1(a), bars were 
installed on both sides of the participants to ensure their safety. A physiotherapist was always close to the participant to manage any unexpected situations. A parameter related to the $\mathrm{CoP}$, the sway area (SA), was collected in order to quantify precisely the sway and give a more accurate representation of the brain's ability to correct balance [37]. The SA was recorded in a simple standing position with eyes closed. The SA is the surface of the ellipse enclosing $90 \%$ of the recorded CoP points [38]. During the two first trials, dedicated to the recording of the SA, participants were instructed to keep the standing position with eyes closed and their arms alongside their body during 60 seconds. To assess the efficacy of the closed-loop control law in both directions, participants underwent 2 trials in the forward closedloop (FCL) condition and 2 trials in the backward closed-loop (B_CL) condition. The order of trials was randomized for the closed-loop conditions.

\section{Instructions given to the participants before each closed-loop trial}

For both F_CL and B_CL conditions, participants were instructed to stand on the force plate, remain still, lightly touch the belt and close their eyes. Before each closed-loop trial, participants were told to: "Stand on this plate, put your index finger on this white tape, look at in front of you and then close your eyes. Remain as still as possible during the trial. The trial will last less than 2 minutes. I will tell you when the trial is finished so you can open your eyes and remove your finger." Closing the eyes was expected to favor the use of the kinesthetic sensory information. Participants were asked to put the tip of their forefinger on a double-sided adhesive tape stuck on the flexiforce sensor before the beginning of the trial and maintain the contact between their finger and the belt for the duration of each trial (as seen in Fig. 1(b)). The physiotherapist verified visually that the participants' forefinger stayed in contact with the belt during the trials. If the participant exceeded a force of $1 \mathrm{~N}$ on the flexiforce sensor, an alarm sound was emitted. The subject was then asked to release the pressure on the belt while maintaining the contact between the sensor and his finger. After the end of the trial ( 80 seconds), participants were allowed to remove their finger from the sensor until the beginning of the next trial. PS patients and controls were asked to use the forefinger of their unaffected side or of their dominant hand respectively.
Participants were not informed of the precise goal of the study. More specifically, they were not aware of the motion of the belt. However, if a participant detected that the belt was moving, the investigators neither confirmed nor denied their suspicions.

\section{Statistical analysis}

Because of the small sample size, scores are reported as medians, first and third quartile values [Q1 - Q3] and the tests are non-parametric.

A non-parametric Mann-Whitney test was used to compare quantitative clinical characteristics and sway area between the two groups. A Chi-square test was used to find any difference in the sex ratio between the two groups.

A paired Wilcoxon signed rank test was run to test any significant differences between corresponding scores from the F_CL and B_CL conditions in each participant.

A non-parametric Mann-Whitney test was used to compare closed-loop parameters $\left(\Delta t_{\text {sat }}, e_{\text {start }}\right.$ and $\left.\epsilon\right)$ between groups.

$\Delta t_{\text {sat }}$ was analysed in all closed-loop trials, whereas $e_{\text {start }}$ and $C$ were only analyzed in retained closedloop trials (with a saturation time lower than the third quartile of $\left.\Delta \mathrm{t}_{\mathrm{ALL}}\right)$.

In PS patients, correlations between the balance scores (Berg score and SA) and the closed-loop parameters $\left(\Delta t_{\text {sat }}, e_{\text {start }}\right.$ and $\left.C\right)$ were determined using the Spearman's correlation test.

All statistical analyses were performed using Graphpad prism software. The statistical level of significance was set at $\mathrm{p}=0.05$.

\section{COMMENTED RESUltS}

All participants completed the 2 trials dedicated to record the sway area and the 4 trials in closed-loop conditions except 1 PS patient who only completed 2 closed-loop trials due to fatigue (one trial in F_CL condition and one trial in $\mathrm{B}$ CL condition). Two trials in F_CL condition (one for a control subject and one for a PS participant) were not recorded because of technical problems.

\section{A. Balance assessment with BERG and posturographic parameters}

As seen in Table 1, post-stroke patients and agedmatched controls were significantly different concerning their Berg score and their sway area. PS patients had significantly worse balance abilities 
than controls with lower BERG scores $(\mathrm{p}<0.001)$ and higher SA $(\mathrm{p}=0.003)$.

B. No difference between the forward and the backward conditions

As seen in Fig. 4, there were no statistically significant differences in $\Delta t_{\text {sat }}(\mathrm{p}=0.18), e_{\text {start }}$ $(\mathrm{p}=0.84)$ and $C(\mathrm{p}=0.96)$ between the F_CL and the B_CL conditions. Therefore, we merged scores obtained between the 2 conditions (F_CL and B_CL). This result indicates that we did not find any difference in the effects of the direction of the proposed closed-loop control.

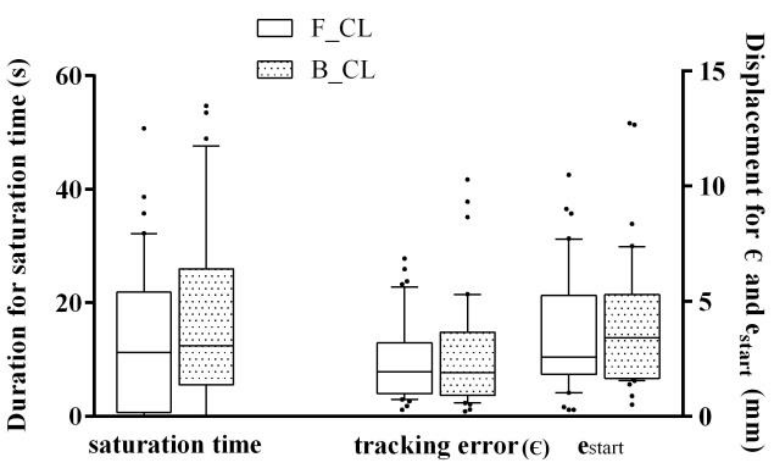

Figure 4: Closed-loop scores in forward (white plot) and backward (dotted plot) conditions. F_CL: forward condition, B_CL: backward condition, $C$ : tracking error. The saturation time values are read on the left $\mathrm{Y}$-axis whereas the tracking error $(\epsilon)$ values and $e_{\text {start }}$ values are read on the right $\mathrm{Y}$-axis. Whisker ends represent the 10 and 90 percentiles.

\section{Saturation time}

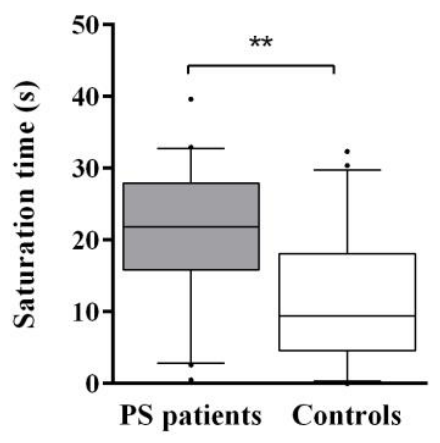

Figure 5: Saturation time in PS patients and controls. Whisker ends represent the 10 and 90 percentiles. PS: post-stroke, ${ }^{* *}: \mathrm{p}<0.01$.

As illustrated in Fig. 5, patients spent significantly more time than controls in the saturation condition $(p=0.006)$. During a closed-loop trial, the participants found themselves in the saturation condition for 9.4 [4.6 - 18.1] seconds and 21.8 [15.8 - 27.9] seconds for the controls and PS patients, respectively.
Moreover, when closed-loop trials from all participants were merged, the median $\Delta \mathrm{t}_{\mathrm{ALL}}$ was 12.4 seconds, corresponding to $\approx 20 \%$ of the total duration of closed-loop control trials. The values of the first and the third quartiles were 0 and 25.5 seconds, respectively. Trials with a $\Delta t_{\text {sat }}$ lower than $25.5 \mathrm{~s}$ for PS and controls were retained for the subsequent analyses of $e_{\text {start }}$ and $\epsilon$. In total, $68 \%$ and $83 \%$ of the closed-loop trials were retained for the PS and control group, respectively.

\section{Closed-loop scores in retained trials $\left(\Delta t_{\text {sat }}<\right.$} $25.5 s$ )

Scores in the closed-loop conditions for the retained trials are illustrated in Fig. 6

The mean tracking error $C$ was similar between groups (controls: 1.4 [0.8 - 1.7], PS: 1.8 [1.3 - 2], $\mathrm{p}=0.06)$. $e_{\text {start }}$ was significantly lower in controls than in patients (controls: 2.2 [1.6 - 2.6], PS: 3.2 [1.8 5.0], $\mathrm{p}=0.04)$, which means that the CoP displacement in response to the closed-loop control was higher in controls than in PS patients.

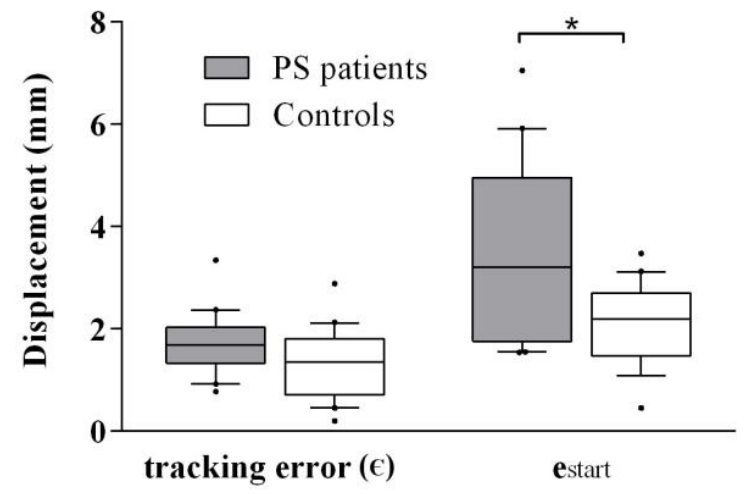

Figure 6: Efficacy scores for the closed-loop paradigm in PS patients and controls in remaining trials. PS: post-stroke, $*: p<0.05$. Whisker ends represent the 10 and 90 percentiles

E. Correlation between balance and closed-loop efficacy scores in PS patients

In PS patients, no significant correlation was found between BERG score and $\epsilon$ (rho $=-0.37,95 \% \mathrm{CI}[-$ $0.70 ; 0.08], \mathrm{p}=0.10), e_{\text {start }}(\mathrm{rho}=-0.16,95 \% \mathrm{CI}[-0.58$ ;0.31], $\mathrm{p}=0.49)$ or $\Delta t_{\text {sat }}(\mathrm{rho}=0.03,95 \% \mathrm{CI}[-0.42$; $0.47], \mathrm{p}=0.90)$.

No significant correlation was found between the sway area and $\epsilon$ (rho $=0.36,95 \% \mathrm{CI}[-0.11 ; 0.70]$, $\mathrm{p}=0.12), e_{\text {start }}($ rho $=0.18,95 \%$ CI $[-0.30 ; 0.59]$, $\mathrm{p}=0.45)$ or $\Delta t_{\text {sat }}($ rho $=-0.26,95 \%$ CI $[-0.64 ; 0.22]$, $\mathrm{p}=0.27$ ). 
The effects of the closed-loop control was independent from the balance impairment severity.

\section{F. Summary of results}

As expected, PS patients had worse balance than aged-matched healthy subjects. As per our first objective, it was demonstrated that the three closedloop scores were similar between the forward and the backward condition. Second, it has been shown that the time spent in saturation condition was statistically greater for PS patients. This led us to discard some trials and showed that the saturation time criteria can discriminate successful trials from unsuccessful ones before even computing $e_{\text {start }}$ and $\epsilon$. The third objective was to assess and compare the closed-loop effects between groups. For the retained trials, there was no significant difference between the groups for the tracking error $C$. However, a slight difference was observed between the two groups for the $\mathrm{e}_{\text {start }}$ criterion.

\section{GeNERAL DISCUSSION}

The results of this study respond to our objectives.

A. The obtained performances of the CoP closedloop control

As expected, the closed-loop control law induced a predefined CoP displacement in PS patients and aged-matched controls in the sagittal plane. This displacement was observed in both directions. These results are therefore encouraging and we stress the fact that participants were naïve about the expected postural changes coupled to the finger displacements. The closed-loop control of the belt fully induced the CoP displacements without the participants' active cooperation. Even if some participants had noticed belt or finger motions, they were not aware of the existing coupling between their finger displacements and their postural sway. Keeping the participants naïve has led us to assess the spontaneous response to the closed-loop paradigm. Our closed-loop control is an unconscious feedback serving to adjust the postural state according to a reference trajectory.

A biofeedback which functions without the active involvement of the user could be particularly useful in patients with important cognitive impairments. No patient included in this study had severe cognitive impairments. It would be interesting to assess in future studies the cognitive functions and to check the effects of our implicit closed-loop control in such population.

The success of the closed-loop is proof that the coupling between finger motion and $\mathrm{CoP}$ displacement, shown in [32], [39], functions in older adults and PS patients. This proof is given here for the first time.

We are now going to focus more specifically on the performances of the proposed closed-loop. The performances were assessed using three scores: the saturation time, $C$ and $e_{\text {start }}$. PS patients showed larger saturation times. However, for the retained trials, both groups performed similarly, even with a slight difference when focusing on the $e_{\text {start }}$ score.

1) The slightly different effects of the closed-loop between post-stroke and healthy subjects

PS patients showed larger saturation times and a slight difference in $e_{\text {start }}$.

One could infer that the haptic and kinesthetic information provided by the belt motion is less clear in patients with more pronounced postural impairment. Indeed, larger postural sway could cause more frequent finger displacement. To study this assumption, we computed the correlation between balance scores and closed-loop sores in PS participants. No correlation was found, meaning that the closed-loop control can drive the CoP in patients experiencing mild to moderate balance impairments. It is worth noting that the ability to maintain a standing posture with no support was a condition for inclusion to the study. This implies that patients with more severe balance impairments, unable to maintain a standing position without aid (human or technical) could not be included. Controlling the CoP of patients with severe balance impairments is not relevant at least in a standing position.

The larger saturation time and the slight difference in $e_{\text {start }}$ could also be attributed to the pathological effects of stroke. First, in post-stroke patients, the sensorimotor function of the unaffected upper extremity is different to that of healthy subjects. Slight impairments are frequent [40]-[42]. The lack of a complete and sound kinesthesia and tactile feedback could contribute to greater saturation durations in such patients. Second, post stroke patients manifest different postural control strategies. It has been reported that post stroke patient actively compensate for their balance impairments [43]. This may explain why coupling between the finger and CoP motions was sometimes 
weaker in PS patients. One could also infer that PS patients could be less confident when moving their $\mathrm{CoP}$ far from their resting position. The maximal displacement in the AP direction, measured during the functional reach test, is lower in post-stroke patients than in healthy subjects, suggesting that post-stroke patients are less confident to move far from their initial position [44].

To sum up, the coupling between the belt and the $\mathrm{CoP}$ displacements functions but is quite less effective in PS patients probably because of minor sensorimotor impairments of the unaffected arm and specific postural strategies.

2) The overall similar effects of the closed-loop between post-stroke and healthy subjects

When considering the tracking error $C$ in the retained trials, the results obtained in post-stroke and agedmatched healthy adults extend those obtained in healthy young people [34], [35]. The tracking error score $(1.05 \pm 1.11 \mathrm{~mm})$ found by Vérité et al. in young healthy adults was approximately the same as that which was measured here in older adults (around 60 years old) and post-stroke patients. One should notice that in our current study, the shape of the reference trajectory has been smoothed.

The control, i.e the belt velocity is permanently adapted based on the current participant's CoP position. Our closed-loop is thus able to adapt to situational changes. If a given participant has a weaker coupling between his/her finger and CoP position, the closure of the loop may overcome this to a certain extent.

To sum up, we suggest that the similarity between the two groups concerning the mean tracking error highlights the efficacy of the closed-loop control to drive the $\mathrm{CoP}$ in a predefined reference trajectory.

\section{B. Improving the performances towards an individually tailored closed-loop tuning}

Even if the closed-loop was effective to drive the $\mathrm{CoP}$, we must admit that some measures could be taken to improve the robustness of the proposed closed-loop paradigm and its effects.

In our study, the same closed-loop gain $\mathrm{K}$ was used for controls and patients. Its value had been determined experimentally (unpublished observation). One can argue that its chosen value was inadequate. The tuning of this feedback gain remains an interesting topic and warrants further investigation. Indeed, the gain $\mathrm{K}$ sets the relationship between the $\mathrm{CoP}$ error (difference between the current and the desired CoP positions) and the finger velocity. If $\mathrm{K}$ were set higher, the finger velocity would also be higher for a given $\mathrm{CoP}$ error. However, a higher gain value would have led to larger displacements and would likely have more induced patients into saturation condition. To circumvent this issue, it might be worth adapting the gain for each subject. The tuning of this gain requires deeper understanding of how sensory information is integrated in postural control and how velocities and amplitudes of finger movements can alter/improve the coupling between postural sway and finger movements. We plan to address these topics in future studies.

Moreover, one could propose to adapt the expected displacements length (chosen to be equal to $8 \mathrm{~mm}$ with a transition velocity of $0.8 \mathrm{~mm} / \mathrm{s}$ ) to the participant in order to avoid excessive difficulty upon $\mathrm{CoP}$ position shift. It may be possible to regulate expected displacement lengths according to each individual's balance performance (CoP parameters or Berg balance score) such that $\mathrm{CoP}$ would be displaced less for those with greater balance difficulties. One should start with a low expected displacement, which could be increased according to the patient's progress.

\section{Future investigations of the closed-loop control of the CoP in neurological environment}

We recognize the important variability of the recorded data. Ideally, these results would be further validated by recruiting more participants in future studies. In addition to this, having a more precise assessment of the patients' sensory and motor function of both upper and lower limbs may assist in better interpreting the variability of data.

The mediolateral displacement direction should also be addressed in post-stroke patients who show great weight-bearing asymmetry in the frontal plane in favor of the unaffected leg [2]. The goal will be to implicitly drive the CoP towards the affected side.

Finally, since preliminary results presented in [35] showed that the closed-loop is efficient even when participants' eyes are open, it would be interesting to combine the closed-loop control with visual biofeedback provided e.g. by the Nintendo Wii balance board (Nintendo, Kyoto, Japan) [15], [20]. The goal will be to supply the CNS with multimodal 
implicit and explicit cues to improve posture in different situations.

\section{Conclusions}

In conclusion, a motorized device with a closed-loop control was used to unconsciously displace the CoP of post-stroke patients and aged control subjects around a desired trajectory. By showing relatively similar closed-loop responses between groups, the present experiment has demonstrated the ability of this device in controlling participants' $\mathrm{CoP}$ without any conscious collaboration. This study demonstrates the interest of closed-loop control systems and paves the road for implementing such devices in rehabilitation settings.

\section{REFERENCES}

[1] D. Sturnieks, R. St George, and S. Lord, "Balance disorders in the elderly.," Neurophysiol. Clin., vol. 38, no. 6, pp. 467478, Dec. 2008.

[2] A. C. H. H. Geurts, M. de Haart, I. J. W. W. van Nes, and J. Duysens, "A review of standing balance recovery from stroke," Gait Posture, vol. 22, no. 3, pp. 267-281, Nov. 2005.

[3] M. M. Gardner, M. C. Robertson, and A. J. Campbell, "Exercise in preventing falls and fall related injuries in older people: a review of randomised controlled trials," Br.J.Sports Med., vol. 34, no. 0306-3674, pp. 7-17, Feb. 2000 .

[4] P. Pollock, G. Baer, P. Campbell, P. Ling Choo, A. Forster, J. Morris, V. M. Pomeroy, and P. Langhorne, "Physical rehabilitation approaches for the recovery of function and mobility following stroke (Review)," Cochrane Database Syst. Rev., no. 4, 2014.

[5] A. Yelnik and I. Bonan, "Clinical tools for assessing balance disorders," Neurophysiologie Clinique, vol. 38, no. 6. pp. 439-445, Dec-2008.

[6] P. Sardain and G. Bessonnet, "Forces acting on a biped robot. Center of pressure-zero moment point," IEEE Trans. Syst. Man. Cybern., vol. 34, no. 5, pp. 630-637, 2004.

[7] T. Paillard and F. Noé, "Techniques and Methods for Testing the Postural Function in Healthy and Pathological Subjects," BioMed Research International, vol. 2015. pp. 1-15, 2015.

[8] T. E. Prieto, J. B. Myklebust, R. G. Hoffmann, E. G. Lovett, and B. M. Myklebust, "Measures of postural steadiness: differences between healthy young and elderly adults.," IEEE Trans. Biomed. Eng., vol. 43, no. 9, pp. 956966, Sep. 1996.

[9] O. M. Giggins, U. Persson, and B. Caulfield, "Biofeedback in rehabilitation," J. Neuroeng. Rehabil., vol. 10, no. 1, p. 60, Jun. 2013.

[10] R. E. Barclay-Goddard, T. J. Stevenson, W. Poluha, M. Moffatt, and S. P. Taback, "Force platform feedback for standing balance training after stroke," Cochrane Database Syst. Rev., Oct. 2004.

[11] A. M. De Nunzio, C. Zucchella, F. Spicciato, P. Tortola, C. Vecchione, F. Pierelli, and M. Bartolo, "Biofeedback rehabilitation of posture and weightbearing distribution in stroke: a center of foot pressure analysis.," Funct. Neurol., vol. 29, no. 2, pp. 127-134, 2014.

[12] R. P. S. Van Peppen, M. Kortsmit, E. Lindeman, and G. Kwakkel, "Effects of visual feedback therapy on postural control in bilateral standing after stroke: a systematic review.," J. Rehabil. Med., vol. 38, no. 1, pp. 3-9, Jan. 2006.

[13] M. W. Kennedy, J. P. Schmiedeler, A. D. Striegel, C. R. Crowell, M. Villano, and J. Kuitse, "Enhanced Feedback in Balance Rehabilitation using the Nintendo Wii Balance Board," IEEE 13th Int. Conf. eHealth Networking, Appl. Serv., 2011.

[14] W. Young, S. Ferguson, S. Brault, and C. Craig, "Assessing and training standing balance in older adults: a novel approach 
using the 'Nintendo Wii' Balance Board.," Gait Posture, vol. 33, no. 2, pp. 303-305, Feb. 2011.

[15] C. Franco, A. Fleury, P.-Y. Guméry, B. Diot, J. Demongeot, and N. Vuillerme, "iBalanceABF: a smartphone-based audio-biofeedback balance system," IEEE Trans. Biomed. Eng., vol. 60, no. 1, pp. 211-215, 2013.

[16] A. A. Gopalai and S. M. N. A. Arosha Senanayake, "A wearable real-time intelligent posture corrective system using vibrotactile feedback," IEEE/ASME Trans. Mechatronics, vol. 16, no. 5, pp. 827-834, 2011.

[17] A. A. Gopalai and S. M. N. A. Senanayake, "Assistive vibrotactile biofeedback system for postural control on perturbed surface," $J$. Mech. Med. Biol., vol. 13, no. 01, p. 1350006, Jun. 2013.

[18] M. R. Afzal, S. Pyo, M.-K. Oh, Y. S. Park, and J. Yoon, "Identifying the effects of using integrated haptic feedback for gait rehabilitation of stroke patients," Int. Conf. Rehabil. Robot., pp. 1055-1060, 2017.

[19] M. R. Afzal, H. Y. Byun, M. K. Oh, and J. Yoon, "Effects of kinesthetic haptic feedback on standing stability of young healthy subjects and stroke patients," J. Neuroeng. Rehabil., vol. 12, no. 1, pp. 1-11, 2015.

[20] A. Mirelman, T. Herman, S. Nicolai, A. Zijlstra, W. Zijlstra, C. Becker, L. Chiari, and J. M. Hausdorff, "Audio-Biofeedback training for posture and balance in Patients with Parkinson's disease," J. Neuroeng. Rehabil., vol. 8, no. 1, p. 35, Jun. 2011.

[21] A. Fung, E. C. Lai, and B. C. Lee, "Usability and Validation of the Smarter Balance System: An Unsupervised Dynamic Balance Exercises System for Individuals with Parkinson's Disease," IEEE Trans. Neural Syst. Rehabil. Eng., vol. 26, no. 4, pp. 798806, 2018.

[22] M. Dozza, L. Chiari, F. Hlavacka, A. Cappello, and F. B. Horak, "Effects of linear versus sigmoid coding of visual or audio biofeedback for the control of upright stance," IEEE Trans. Neural Syst. Rehabil. Eng., vol. 14, no. 4, pp. 505-512, 2006.

[23] J. J. Jeka and J. R. Lackner, "Fingertip contact influences human postural control.," Exp. Brain Res., vol. 100, no. 3, pp. 495-502, 1994.

[24] A. M. S. Baldan, S. R. Alouche, I. M. G. Araujo, and S. M. S. F. Freitas, "Effect of light touch on postural sway in individuals with balance problems: A systematic review," Gait Posture, vol. 40, no. 1, pp. 1-10, May 2014.

[25] B. P. Cunha, S. R. Alouche, I. M. G. Araujo, and S. M. S. F. Freitas, "Individuals with poststroke hemiparesis are able to use additional sensory information to reduce postural sway," Neurosci. Lett., vol. 513, no. 1, pp. 6-11, Mar. 2012.

[26] S.-H. Lee, D. Lee, Y. Lee, Y. Jee, G. Lee, and D.-S. Park, "Influence of light touch using the fingertips on postural stability of poststroke patients," J. Phys. Ther. Sci., vol. 27, no. 2, pp. 469-472, Feb. 2015.

[27] J. J. Jeka, "Light Touch Contact as a Balance Aid," Phys. Ther., vol. 77, no. 5, pp. 476-487, May 1997.

[28] I. M. Albertsen, J. J. Temprado, and E. Berton, "Effect of haptic supplementation on postural stabilization: A comparison of fixed and mobile support conditions.," Hum. Mov. Sci., vol. 29, no. 6, pp. 999-1010, Dec. 2010.

[29] R. Dickstein, "Stance stability with unilateral and bilateral light touch of an external stationary object.," Somatosens. Mot. Res., vol. 22, no. 4, pp. 319-325, Dec. 2005.

[30] Y.-T. Pan, H. U. Yoon, and P. Hur, "A Portable Sensory Augmentation Device for Balance Rehabilitation Using Fingertip Skin Stretch Feedback.," IEEE Trans. Neural Syst. Rehabil. Eng., vol. 25, no. 1, pp. 28-36, 2017.

[31] J. J. Jeka, G. Schöner, T. Dijkstra, P. Ribeiro, and J. R. Lackner, "Coupling of fingertip 
somatosensory information to head and body sway," Exp. Brain Res., vol. 113, no. 3, pp. 475-483, Mar. 1997.

[32] A. M. Wing, L. Johannsen, and S. Endo, "Light touch for balance: influence of a timevarying external driving signal," Philos. Trans. R. Soc. B Biol. Sci., vol. 366, no. 1581, pp. 3133-3141, Nov. 2011.

[33] J. Jeka, K. S. Oie, and T. Kiemel, "Multisensory information for human postural control: integrating touch and vision.," Exp. brain Res., vol. 134, no. 1, pp. 107-125, Sep. 2000.

[34] F. Verite, W. Bachta, and G. Morel, "Closedloop control of a human Center-Of-Pressure position based on somatosensory feedback," IEEE Int. Conf. Intell. Robot. Syst., pp. 42554261, 2013.

[35] F. Verite, W. Bachta, and G. Morel, "Closed loop kinesthetic feedback for postural control rehabilitation," IEEE Trans. Haptics, vol. 7, no. 2, pp. 150-160, Jun. 2014.

[36] F. Tremblay, A.-C. Mireault, L. Dessureault, H. Manning, and H. Sveistrup, "Postural stabilization from fingertip contact: I. Variations in sway attenuation, perceived stability and contact forces with aging.," Exp. brain Res., vol. 157, no. 3, pp. 275-285, Aug. 2004.

[37] G. Kim, M. Ferdjallah, and G. F. Harris, "Fast differential analysis of center of pressure data in normal children and children with cerebral palsy," Am. J. Biomed. Sci., vol. 24, no. 4, pp. 341-342, 2009.

[38] P. Schubert and M. Kirchner, "Ellipse area calculations and their applicability in posturography.," Gait Posture, vol. 39, no. 1, pp. 518-522, 2014.

[39] J. J. Jeka, K. Oie, G. Schöner, T. Dijkstra, and E. Henson, "Position and velocity coupling of postural sway to somatosensory drive.," $J$. Neurophysiol., vol. 79, no. 4, pp. 1661-1674, Apr. 1998.
[40] H. Y. Hsu, C. W. Ke, T. S. Kuan, H. C. Yang, C. L. Tsai, and L. C. Kuo, "Impacts of Sensation, Perception, and Motor Abilities of the Ipsilesional Upper Limb on Hand Functions in Unilateral Stroke: Quantifications From Biomechanical and Functional Perspectives," $P M R$, vol. 10, no. 2, pp. 146-153, 2018.

[41] N. Lima, K. Menegatti, É. Yu, N. Sacomoto, T. Scalha, I. Lima, S. da Camara, M. de Souza, R. Cacho, E. Cacho, and D. Honorato, "Sensory deficits in ipsilesional upperextremity in chronic stroke patients," Arq. Neuropsiquiatr., vol. 73, no. 10, pp. 834-839, 2015.

[42] J. Metrot, J. Froger, I. Hauret, D. Mottet, L. van Dokkum, and I. Laffont, "Motor recovery of the ipsilesional upper limb in subacute stroke.," Arch. Phys. Med. Rehabil., vol. 94, no. 11, pp. 2283-2290, Nov. 2013.

[43] M. Roerdink, M. De Haart, A. Daffertshofer, S. F. Donker, A. C. H. Geurts, and P. J. Beek, "Dynamical structure of center-of-pressure trajectories in patients recovering from stroke.," Exp. brain Res., vol. 174, no. 2, pp. 256-269, Sep. 2006.

[44] S. Portnoy, S. Reif, T. Mendelboim, and D. Rand, "Postural control of individuals with chronic stroke compared to healthy participants: Timed-Up-and-Go, Functional Reach Test and center of pressure movement.," Eur. J. Phys. Rehabil. Med., vol. 53, no. 5, pp. 685-693, 2017. 